\title{
openheart Associations between physical activity, sedentary behaviour and left ventricular structure and function from the Echocardiographic Study of Latinos (ECHO-SOL)
}

Andrew E Berdy, ${ }^{1}$ Bharathi Upadhya (D) , ${ }^{1}$ Sonia Ponce, ${ }^{2}$ Katrina Swett, ${ }^{3}$ Richard B Stacey, ${ }^{1}$ Robert Kaplan, ${ }^{3}$ Priscilla M Vasquez, ${ }^{2}$ Qibin Qi, ${ }^{3}$ Neil Schneiderman, ${ }^{4}$ Barry E Hurwitz, ${ }^{4}$ Martha L Daviglus, ${ }^{5}$ Mayank Kansal, ${ }^{5}$ Kelly R Evenson, ${ }^{6}$ Carlos J Rodriguez ${ }^{3}$

- Additional supplemental material is published online only. To view, please visit the journal online (http://dx.doi.org/10. 1136/openhrt-2021-001647).

To cite: Berdy AE, Upadhya B, Ponce S, et al. Associations between physical activity, sedentary behaviour and left ventricular structure and function from the Echocardiographic Study of Latinos (ECHO-SOL). Open Heart 2021;8:e001647. doi:10.1136/ openhrt-2021-001647

Received 11 March 2021 Accepted 15 June 2021

Check for updates

(C) Author(s) (or their employer(s)) 2021. Re-use permitted under CC BY-NC. No commercial re-use. See rights and permissions. Published by BMJ.

For numbered affiliations see end of article.

Correspondence to Dr Bharathi Upadhya; bupadhya@wakehealth.edu

\section{ABSTRACT}

Objective The cross-sectional association between accelerometer-measured physical activity (PA), sedentary behaviour (SB) and cardiac structure and function is less well described. This study's primary aim was to compare echocardiographic measures of cardiac structure and function with accelerometer measured PA and SB. Methods Participants included 1206 self-identified Hispanic/Latino men and women, age 45-74 years, from the Echocardiographic Study of Latinos. Standard echocardiographic measures included M-mode, twodimensional, spectral, tissue Doppler and myocardial strain. Participants wore an Actical accelerometer at the hip for 1 week.

Results The mean \pm SE age for the cohort was $56 \pm 0.4$ years, $57 \%$ were women. Average moderate to vigorous PA (MVPA) was $21 \pm 1.1 \mathrm{~min} /$ day, light PA was $217 \pm 4.2 \mathrm{~min} /$ day and $S B$ was $737 \pm 8.1 \mathrm{~min} /$ day. Both higher levels of light PA and MVPA (min/day) were associated with lower left ventricular (LV) mass index (LVMI)/end-diastolic volume and a lower E/e' ratio. Higher levels of MVPA ( $\mathrm{min} /$ day) were associated with better right ventricular systolic function. Higher levels of SB were associated with increased LVMI. In a multivariable linear regression model adjusted for demographics and cardiovascular disease modifiable factors, every 10 additional min/day of light PA was associated with a $0.03 \mathrm{~mL} / \mathrm{m}^{2}$ increase in left atrial volume index $(\mathrm{LAVI})(p<0.01)$ and a $0.004 \mathrm{~cm}$ increase in tricuspid annular plane systolic excursion $(p<0.01)$; every 10 additional min/day of MVPA was associated with a $0.18 \mathrm{~mL} / \mathrm{m}^{2}$ increase in LAVI $(p<0.01)$ and a $0.24 \%$ improvement in global circumferential strain $(p<0.01)$. Conclusions Our findings highlight the potential positive association between the MVPA and light PA on cardiac structure and function.

\section{INTRODUCTION}

Lack of physical activity (PA) is common in the US population, and it is associated with multiple detrimental health outcomes.

\section{Key questions}

What is already known about this subject?

- Hispanic/Latino are the largest ethnic minority in the USA and represent a high-risk population; higher prevalence of cardiovascular disease factors, relatively understudied population and higher rates of sedentary behaviour (SB) and lower rates of physical activity (PA) than other ethnic groups.

What does this study add?

- To our knowledge, this is the first study to examine the association between moderate to vigorous PA and favourable left ventricle myocardial dynamics, remodelling, improved right ventricle function without any association with left ventricular mass in a large cohort of asymptomatic Hispanics/Latinos.

How might this impact on clinical practice?

- These findings may allow for more detailed, patientspecific, risk-stratification and prognosis in healthy individuals by gaining insight into the interpretation of resting echocardiography. This study provides further evidence that formal recommendations on limiting SB in persons of varying age should be developed to provide public health professionals and clinical practitioners with information to improve their effectiveness in promoting PA and health Physical inactivity should be considered as an important cardiovascular disease risk factor.

Troubling is the even lower trend of PA in Hispanic/Latino men and women. According to 2008 report of the National Health Interview Survey, Hispanics/Latinos had lower leisure-time PA levels than non-Hispanic whites. ${ }^{2}$ Similarly, 2007-2016 National Health and Nutrition Examination Survey (selfreported data) indicate that Hispanics/ Latinos are less likely to meet recommended 
PA guidelines compared with non-Hispanic whites $(33.4 \%$ vs $47.6 \%){ }^{3}$

$\mathrm{PA}$ is associated with cardiovascular (CV) health benefits, including prevention of age-related cardiac remodelling, systolic and diastolic dysfunction, adaptive pro-hypertrophic effect, decreased markers of inflammation, improved metabolic health, decreased risk of heart failure (HF) and improved overall survival. ${ }^{4-8}$ Physical inactivity is associated with obesity, diabetes, hypertension and the metabolic syndrome. ${ }^{9-11}$ All these conditions share several underlying mechanisms that result in increased HF risk; insulin resistance, impaired energy availability, endothelial dysfunction, oxidative stress, which ultimately leads to increased myocardial stiffness, left ventricular (LV) hypertrophy (LVH), diastolic dysfunction and an increased propensity for coronary artery disease. ${ }^{12-14}$

A majority of previous epidemiological studies have been limited by a lack of objective measures on PA and sedentary behaviour (SB). A prior study that used accelerometry data only included a large community-based sample of young adults to middle-aged men and women. ${ }^{67}$ Additionally, the Hispanic/Latino population is understudied, with a limited number of large-scale analyses of this population, particularly those that consider the wide array of ethnic, cultural and geographic diversity.

Echocardiographic Study of Latinos (ECHO-SOL), an ancillary echocardiographic study of the Hispanic Community Health Study/Study of Latinos (HCHS/ SOL), is a multicentre epidemiological study designed to include a diverse Hispanic/Latino subgroup representation and provided a unique opportunity to explore the cross-sectional association between accelerometermeasured $\mathrm{PA}, \mathrm{SB}$, and cardiac structure and function as measured by transthoracic echocardiography (TTE). This study's primary aim was to compare echocardiographic measures of cardiac structure and function with accelerometer measured PA and SB. We hypothesised that higher PA would be favourably associated, and SB would be negatively associated with LV structure and function.

\section{METHODS \\ Study setting}

HCHS/SOL is a prospective population-based longitudinal cohort study $(n=16,415$, mean age 43 years $)$ of self-identified Hispanic/Latino individuals, 18-74 years of age enrolled from 2008 to $2011 .{ }^{15}$ This study was designed to assess chronic disease in Hispanic/Latino individuals living in four cities in the USA: Bronx, New York; Chicago, Illinois; Miami, Florida; and San Diego, California. Participants were recruited in communities surrounding four field centres in the respective cities. The details of the HCHS/SOL sampling method have been previously described. ${ }^{15}$

ECHO-SOL is an ancillary study of HCHS/SOL consisting of 1818 participants recruited through a stratified sampling process representative of the parent study. ${ }^{16}$ ECHO-SOL was designed to characterise cardiac structure and function using TTE in a representative sample of Hispanic/Latino individuals living in the USA. The baseline TTE examination for the ECHO-SOL ancillary study was performed from 2011 to $2014 .{ }^{16}$ Inclusion criteria for ECHO-SOL included: age 45 years or older; self-reported Hispanic/Latino of Mexican, Puerto Rican, Cuban, Dominican, Central American or South American background; and enrolment 36 months or fewer from the date of the baseline HCHS/SOL visit. ECHO-SOL enrolled, on average, $\sim 80 \%$ of eligible participants at each of the study sites. Inclusion and exclusion criteria for ECHO-SOL was previously described. ${ }^{16} 17$

The current analysis excluded participants who did not wear the accelerometer or were not adherent to the accelerometer protocol $(\mathrm{n}=465$; at least 3 days with 10 hours of wear time each). We excluded those with poor quality data on at least one echocardiographic variable $(n=39)$ and participants with prior CV disease (CVD) for our analysis $(n=108)$. These exclusions yielded an analytic sample of $n=1206$ ( $66 \%$ of participants). The baseline characteristics of all 1818 ECHO-SOL participants were previously described. ${ }^{17}$

\section{STUDY MEASURES \\ PA measures}

The Actical accelerometer (version B-1 model 198-020003; Respironics Co, Bend, Oregon) was used to assess PA and SB. The accelerometer data were collected at the baseline examination visit. During the HCHS/SOL baseline clinic visit, participants were fitted with a belt and left the clinic wearing the accelerometer above the iliac crest. Participants were instructed to perform normal daily activities and wear the accelerometer only during awake hours and remove it for sleep, showering and swimming. The Actical device was programmed to measure accelerations in counts per 1 min epochs based on the convention used in previous studies. ${ }^{18}$ The time used for analysis started at 05:00 the morning after the initial clinic visit and ended at midnight on the sixth day of wear. Nonwear was defined as consecutive zero counts for at least 90 min. ${ }^{18}$ PA threshold levels were defined as follows: SB $<100$ counts/min, light 100-1534 counts/min, moderate 1535-3961 counts/min and vigorous $\geq 3962$ counts/ min. ${ }^{19}{ }^{20}$ For the present analysis, data for moderate to vigorous PA (MVPA) were combined, denoted as MVPA, because very few people had a significant amount of vigorous activity. Adherence and accelerometer performance have been previously described. ${ }^{18}$ Adherence was defined as $\geq 10$ hours/day of wear time for at least 3 days of wear. Time spent in PA by intensity level was calculated by summing the minutes in a day where the counts were within each threshold and then averaging across adherent days.

The HCHS/SOL assessed self-reported PA in a typical week using an interviewer-administered, modified Global 
PA Questionnaire that included questions on three activity domains (work, transport and leisure) available at www.int/chp/steps/GPAQ/en. Participants were asked to think about activities that lasted at least $10 \mathrm{~min}$ in a typical week. We only included accelerometer-measured PA for our analysis.

\section{Echocardiography measures}

The average time between the accelerometer data and echocardiographic measures was $815 \pm 196$ days. A complete description of the echocardiographic measures of cardiac structure and function was previously reported. ${ }^{161721}$ Briefly, a standardised TTE ultrasound examination, including M-mode, two-dimensional, spectral, colour flow and tissue Doppler, was performed by experienced Registered Diagnostic Cardiac Sonographers at each of the four parent study field sites using Philips IE-33 or Sonos 7500 scanners interfaced with a standard 2.5-3.5 MHz phased-array probe, according to American Society of Echocardiography (ASE) recommendations. ${ }^{22}$ Echocardiograms were analysed and interpreted centrally at Wake Forest School of Medicine (Winston-Salem, North Carolina, USA). Inter-reader and intra-reader reproducibility was assessed and previously reported. ${ }^{16}$ Two-dimensional imaging of the LV was performed to obtain the best possible images of the LV endocardium without foreshortening of the LV cavity or echo drop out. Using the apical 4-chamber and 2-chamber views, LV enddiastolic volumes (EDV) and end-systolic volumes (ESV) were derived using the biplane method of discs, as per the ASE recommended methodology. ${ }^{22}$ The following echocardiographic measured and derived variables were used: LV mass (LVM) was determined by subtracting the LV endocardial cavity volume from the LV epicardial volume and multiplying the resultant myocardial volume by the myocardial density $(1.05 \mathrm{~g} / \mathrm{mL})$ then indexing based on the body surface area or height ${ }^{2.7}$. LV geometry was predicated on the measurement of LVM, LV posterior wall thickness at diastole (LVPWd), interventricular septal thickness at diastole and relative wall thickness (RWT) defined as $2 \times(\mathrm{LVPWd} / \mathrm{LV}$ internal diameter at diastole). LV geometry was defined as normal (normal LVM and normal RWT) or abnormal with abnormal LV geometry categorised as follows: concentric remodelling (normal LVM and increased RWT), eccentric hypertrophy (increased LVM and normal RWT) and concentric hypertrophy (increased LVM and increased RWT) ${ }^{22}$ $\mathrm{LV}$ ejection fraction $(\mathrm{LVEF})=(\mathrm{EDV}-\mathrm{ESV}) / \mathrm{EDV}$. LVEF could not be ascertained in $4.9 \%$ of the cohort because of image quality. Our assessment of LV diastolic dysfunction (LVDD) has been previously published. ${ }^{21}$ Right ventricular (RV) systolic function and tricuspid valve regurgitation velocity (TRV) were assessed according to ASE guidelines. Speckle-tracking strain analysis was performed offline using the TomTec Cardiac Performance Analysis package on two-dimensional images acquired, and methods were previously described. ${ }^{16}$
Primary structural and functional measures included LVM indexed to height ${ }^{2.7}$ (LVMI), RWT, left atrial volume (LAV), LAV index (LAVI) and EF, mid-LV segment global circumferential strain (GCS), global longitudinal strain (GLS) and LV diastolic functional measures. Secondary cardiac measures included LV stroke volume (LVSV), lateral peak systolic annular velocity (lat peak S'), RV fractional area change (RV FAC), TRV, tricuspid annulus systolic velocity (RV S' wave), RV velocity-time integral (RV VTI), RVSV and tricuspid annular plane systolic excursion (TAPSE).

\section{Clinical covariates}

All measures were taken from the baseline visit (20082011). Trained technicians measured each participant's height and weight twice and then averaged these two measures to calculate body mass index (BMI=weight $(\mathrm{kg}) /$ height $\left.\left(\mathrm{m}^{2}\right)\right)$. Participants with a BMI of $\geq 30 \mathrm{~kg} /$ $\mathrm{m}^{2}$ were categorised as obese. Three seated blood pressure (BP) measures were taken using an automatic sphygmomanometer averaging the last two measures. Hypertension was defined as a systolic BP of $140 \mathrm{~mm}$ $\mathrm{Hg}$ or greater, diastolic BP of $90 \mathrm{~mm} \mathrm{Hg}$ or greater, or receiving antihypertensive medication. Participants were classified as having hypercholesterolaemia if they were currently using the cholesterol-lowering medication, had low-density lipoprotein levels $\geq 160 \mathrm{mg} / \mathrm{dL}$, or total cholesterol $\geq 240 \mathrm{mg} / \mathrm{dL}$. Total and high-density lipoprotein (HDL) cholesterol was measured with a direct magnesium/dextran sulfate method. Plasma glucose was measure fasting and after a 2-hour glucose load. Diabetes mellitus was a fasting plasma glucose of $126 \mathrm{mg} /$ dL or greater, 2-hour-post load glucose of $200 \mathrm{mg} / \mathrm{dL}$ or greater, glycosylated haemoglobin $6.5 \%$ or greater, or use of antihyperglycaemic medications. Self-reported current smoking history referring to cigarette smoking and current alcohol intake referring to alcohol history was collected via questionnaire. ${ }^{23}$

Healthy subgroup $(n=460)$ was defined as not having hypertension (systolic $\mathrm{BP} \geq 140 \mathrm{~mm} \mathrm{Hg}$, diastolic $\mathrm{BP} \geq 80 \mathrm{~mm} \mathrm{Hg}$ or history of drug-treated hypertension), diabetes (history of a diagnosis of diabetes mellitus, fasting glucose $\geq 126 \mathrm{mg} / \mathrm{dL}$ or glycosylated haemoglobin $\geq 6.5 \%$ ), coronary heart disease (self-reported history of revascularisation, myocardial infarction, angina or ECG defined evidence of myocardial infarction) or obesity $\left(\mathrm{BMI} \geq 30 \mathrm{~kg} / \mathrm{m}^{2}\right)$.

\section{Statistical methods}

The primary aim of this study was to compare echocardiographic measures of cardiac structure and function in participants with higher levels of PA and SB. The dependent variables were echocardiographic measures of cardiac structure and function. SB, light PA and MVPA were the primary independent variables. Cohort characteristics at baseline were described using means and SEs for continuous variables and frequencies (percentages) for categorical variables. $\mathrm{SB}$, light PA and MVPA, and all echocardiographic measures were treated as continuous variables. 
Hypertension, diabetes and current cigarette smoking were treated as categorical variables. The means of echocardiographic measures of cardiac structure and function were compared across categories of PA (MVPA, light $\mathrm{PA}$ ) and SB using multivariable linear regression models. Age, sex, hypertension, antihypertensive medications, diabetes, current cigarette smoking and HDL cholesterol were included as covariates in the analysis. Covariates were chosen based on known associations with ventricular size and heart disease, including demographics and anthropometric variables, and variables reflecting comorbidities. Model 1-demographics, model 2-model 1 plus continuous systolic BP and antihypertension medication use, model 3-model 2 plus other CV disease modifiable factors (diabetes mellitus, current smoking and continuous HDL). We divided the light PA, MVPA and SB into quartiles (Q1-Q4) and compared the echocardiographic variables between the highest quartile $(\mathrm{Q} 4)$ to other quartiles by univariate analysis.

Estimated beta coefficients and $p$ values based on the $F$ statistic were presented. Statistical significance was designated as a $p$ value $\leq 0.01$ with no adjustments for multiple comparisons. SAS SURVEY procedures were used, and all reported values were weighted to adjust for sampling probability and non-response bias to make the inference applicable to the ECHO-SOL target population. All analyses were performed using SAS V.9.4 (SAS Institute).

\section{RESULTS}

Baseline characteristics of the study population are provided in table 1 . The mean age of included participants was 56 years; $57 \%$ were women, $28 \%$ had diabetes, $50 \%$ had hypertension and $18 \%$ reported current cigarette smoking. On average, participants accumulated (mean $\pm \mathrm{SD}$ ) $21 \pm 1.1 \mathrm{~min} /$ day of MVPA; $217 \pm 4.2 \mathrm{~min} /$ day of light PA and $737 \pm 8.1 \mathrm{~min} /$ day of SB. Men accumulated more MVPA compared with women $(27 \pm 1.6$ vs $17 \pm 1.3 \mathrm{~min} /$ day, $\mathrm{p}<0.001$ ) (figure 1 ). Participants aged $>65$ years had less MVPA accumulation than participants aged $<55$ years $(17 \pm 3.0$ vs $24 \pm 1.5, p=0.03)$. Overall, participants aged $>55$ years spent less time in both light PA and MVPA than those less than 55 years of age (figure 2). Online supplemental table 1 shows the prevalent selfreported PA in ECHO/SOL.

\section{Associations between PA and echocardiographic measures Light PA}

Compared with less light PA time (quartile 1), more light PA (quartile 4) was associated with decreased LVMI/ EDV ratio $(\mathrm{p}<0.001)$ and a decrease in $\mathrm{E} / \mathrm{e}^{\prime}$ ratio $(\mathrm{p}<0.001)$ (online supplemental table 2). In multivariable linear regression models between light PA and echocardiographic measures (model 3), every $10 \mathrm{~min} /$ day of light PA was associated with a $0.03 \mathrm{~mL} / \mathrm{m}^{2}$ increase in LAVI $(\mathrm{p}<0.01)$ and a $0.004 \mathrm{~cm}$ increase in TAPSE $(p<0.01)$. There were no statistically significant associations between light PA and other echocardiographic measures (table 2).
Table 1 Baseline characteristics

\begin{tabular}{|c|c|}
\hline Variables & $n=1206$ \\
\hline Age, years & $56.4 \pm 0.36$ \\
\hline Female, \% & $691(57)$ \\
\hline Body mass index $\left(\mathrm{kg} / \mathrm{m}^{2}\right)$ & $30.1(0.1)$ \\
\hline Body surface area $\left(\mathrm{m}^{2}\right)$ & $1.8(0.1)$ \\
\hline Systolic BP, mm Hg & $136(1.0)$ \\
\hline Diastolic BP, mm Hg & $78(0.7)$ \\
\hline Diabetes, \% & $343(28)$ \\
\hline Hypertension, \% & $603(50)$ \\
\hline Hypercholesterolaemia, \% & $626(49)$ \\
\hline HDL cholesterol, mg/dL & $50.4(0.7)$ \\
\hline Alcohol use, \% & $903(75)$ \\
\hline Current cigarette smoking, $\%$ & $212(18)$ \\
\hline \multicolumn{2}{|l|}{ Echocardiographic measurements } \\
\hline Left ventricular mass index (LVMI, $\mathrm{g} / \mathrm{m}^{2.7}$ ) & $41.5(0.35)$ \\
\hline Relative wall thickness, ratio & $0.40(0.003)$ \\
\hline $\mathrm{LVMI}^{2.7}$ /end-diastolic volume & $0.52(0.006)$ \\
\hline Left atrial volume index, $\mathrm{mL} / \mathrm{m}^{2}$ & $23.02(0.25)$ \\
\hline Left ventricular ejection fraction, $\%$ & $59.80(0.20)$ \\
\hline$E / e^{\prime}$, ratio & $9.96(0.12)$ \\
\hline Global longitudinal strain, \% & $-17.56(0.13)$ \\
\hline Global circumferential strain, $\%$ & $-24.33(0.29)$ \\
\hline LV stroke volume, $\mathrm{mL}$ & $70.30(0.61)$ \\
\hline Lateral peak S velocity, $\mathrm{cm} / \mathrm{s}$ & $8.30(0.08)$ \\
\hline RV peak S velocity, $\mathrm{cm} / \mathrm{s}$ & $11.88(0.09)$ \\
\hline RV fractional area change, $\%$ & $0.45(0.004)$ \\
\hline TR max velocity, $\mathrm{cm} / \mathrm{s}$ & $206.35(1.69)$ \\
\hline RV velocity-time integral, $\mathrm{cm}$ & $17.42(0.15)$ \\
\hline TAPSE, cm & $2.09(0.02)$ \\
\hline \multicolumn{2}{|l|}{ Physical activity and sedentary behaviour } \\
\hline MVPA, min/day & $20.93 \pm 1.1$ \\
\hline Light PA, min/day & $216.84 \pm 4.2$ \\
\hline Sedentary behaviour, min/day & $736.50 \pm 8.1$ \\
\hline
\end{tabular}

$\mathrm{N}$ (\%) using weighted row percentages; N's presented are weighted counts.

Data presented in means \pm SE of mean.

$\mathrm{BP}$, blood pressure; E, peak early diastolic transmitral inflow velocities; $e^{\prime}$, average of mitral early diastolic septal and lateral annular velocities; HDL, high-density lipoprotein; LV, left ventricular; MVPA, moderate to vigorous-intensity PA; $n$, number; PA, physical activity; RV, right ventricular; TAPSE, tricuspid annular plane systolic excursion; TR, tricuspid regurgitation.

\section{Moderate to vigorous PA}

Compared with less MVPA (quartile 1), greater MVPA (quartile 4) was associated with decreased LVMI/EDV ratio $(p<0.001)$, increased LAVI $(p<0.001)$ and decrease in $\mathrm{E} / \mathrm{e}^{\prime} \quad(\mathrm{p}<0.001)$ ratio (online supplemental table $3)$. In a multivariable linear regression model between PA and echocardiographic measures (model 3), every 


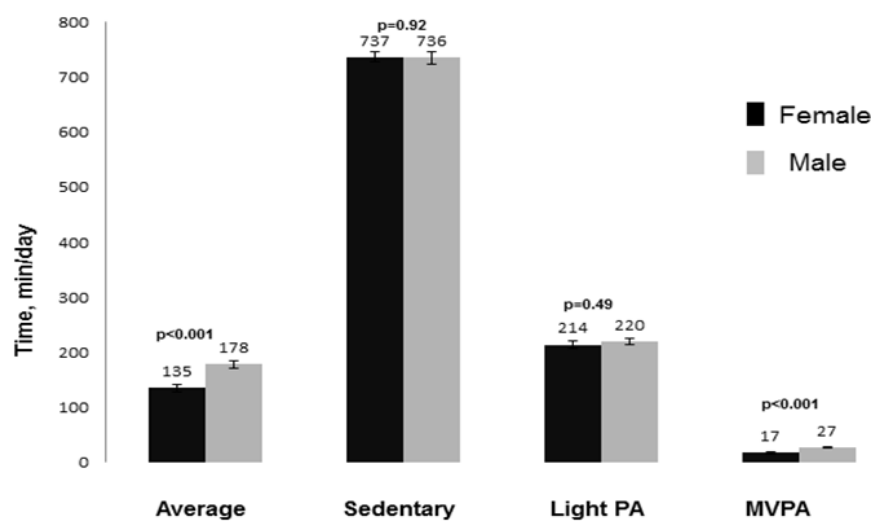

Figure 1 Physical activity and sedentary behaviour by gender. ${ }^{*}$ Data presented in means \pm SE of mean. The number above the bar shows mean activity in min/day. MVPA, moderate to vigorous-intensity PA; PA, physical activity.

$10 \mathrm{~min} /$ day of MVPA was associated with a $0.18 \mathrm{~mL} / \mathrm{m}^{2}$ increase in LAVI $(\mathrm{p}<0.01)$ and a $0.24 \%$ improvement in GCS $(\mathrm{p}<0.01)$. No statistically significant associations were noted between MVPA and other echocardiographic measures (table 3).

\section{SB and echocardiographic measures}

Compared with lower SB (quartile 1), higher SB (quartile 4) was associated with increased LVMI $(\mathrm{p}=0.008)$ (online supplemental table 4). In multivariable linear regression models between SB and echocardiographic measures (model 3), there were no statistically significant associations between SB and any of the echocardiographic measures (table 4). Online supplemental table 5 shows an unadjusted association between PA and echocardiographic measures.

\section{PA and echocardiographic measures in healthy subgroup}

In a multivariable linear regression model between PA and echocardiographic measures (model 3), both MVPA and light PA was associated with increased LAVI, improved GLS, improved $\mathrm{E} / \mathrm{e}$ ratio, improved RV function (increase in TAPSE); MVPA was associated

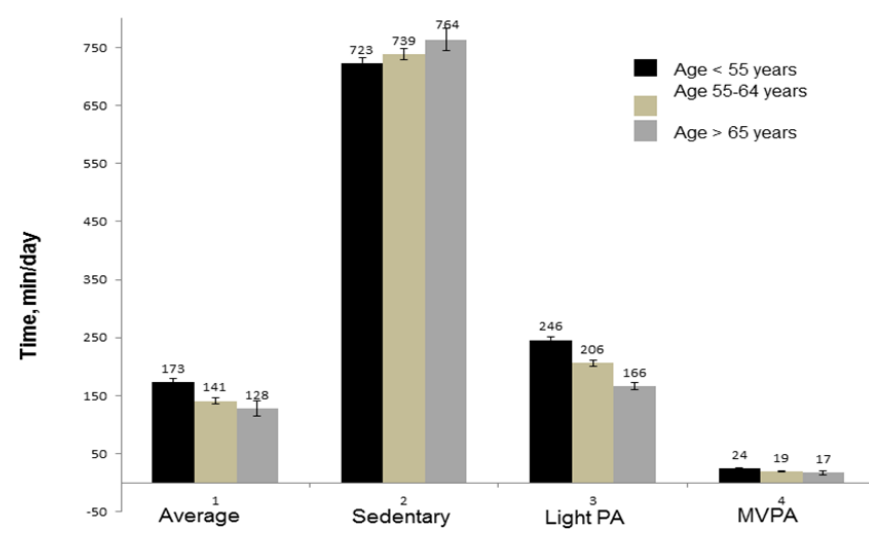

Figure 2 Physical activity and sedentary behaviour by age. Data presented in means \pm SE of mean. The number above the bar shows mean activity in min/day. MVPA, moderate to vigorous-intensity PA; PA, physical activity.
Table 2 Multivariable linear regression for the association between light PA and echocardiogram parameters

\begin{tabular}{|c|c|c|c|}
\hline Outcome & Model 1 & Model 2 & Model 3 \\
\hline LVMI, $\mathrm{g} / \mathrm{m}^{2.7}$ & -0.02882 & -0.00597 & 0.01003 \\
\hline $\mathrm{LVMI}^{2.7} / \mathrm{EDV}$ & -0.00157 & -0.00135 & -0.00108 \\
\hline RWT, ratio & 0.00014 & 0.00021 & 0.00030 \\
\hline LAVI, $\mathrm{mL} / \mathrm{m}^{2}$ & $0.02431^{*}$ & $0.03206^{*}$ & $0.02840^{x}$ \\
\hline $\mathrm{EF}, \%$ & 0.01336 & 0.01369 & 0.00728 \\
\hline E/e', ratio & -0.00843 & -0.00521 & 0.00086 \\
\hline GLS, $\%$ & -0.02085 & -0.01784 & -0.01333 \\
\hline GCS, \% & -0.02332 & -0.02139 & -0.01778 \\
\hline $\mathrm{SV}, \mathrm{mL}$ & -0.02847 & -0.03209 & -0.03206 \\
\hline $\mathrm{RV}$ peak $\mathrm{S}, \mathrm{cm} / \mathrm{s}$ & 0.01126 & 0.01249 & 0.01287 \\
\hline RV FAC, \% & 0.00048 & 0.00051 & 0.00034 \\
\hline TRV Max, cm/s & 0.20262 & 0.18327 & 0.13431 \\
\hline TAPSE, cm & $0.0037^{*}$ & $0.0040^{*}$ & $0.0037^{*}$ \\
\hline
\end{tabular}

M1: demographics (age and sex).

M2: M1+systolic blood pressure, antihypertensive medication use. M3: M2+other cardiovascular disease modifiable factors (diabetes mellitus, current smoking and continuous high-density lipoprotein). Values are per $10 \mathrm{~min} /$ day light PA.

${ }^{*} \mathrm{P}$ value $<0.01$.

E, peak early diastolic transmitral inflow velocities; EDV, end diastolic volume; $\mathrm{e}^{\prime}$, average of mitral early diastolic septal and lateral annular velocities; EF, ejection fraction; GCS, global circumferential strain; GLS, global longitudinal strain; LAVI, left atrial volume index; LVMI, left ventricular mass index; M, model; PA, physical activity; RV, right ventricular; RV FAC, RV fractional area change; RWT, relative valve thickness; SV, stroke vol; TAPSE, tricuspid annular plane systolic excursion; TRV, tricuspid regurgitation velocity.

with greater SV (online supplemental table 6). SB was associated with worsening E/e, GLS and RV function (decrease in TAPSE). SB was negatively correlated with SV.

We performed additional analysis to find the correlation between MVPA and SB. The correlation between sedentary time and MVPA is very weak with a Pearson correlation coefficient of $-0.1(\mathrm{p}=0.26)$ and a very flat slope on the scatterplot of the two variables.

\section{DISCUSSION}

In this large cohort of diverse Hispanic/Latinos, we found that higher light PA was associated with increased LAV and better RV systolic function after adjustment for potential confounders. Similarly, higher MVPA was associated with greater LV myocardial mechanics as assessed by strain and increased LAV. Both higher levels of light PA and MVPA ( $\mathrm{min} /$ day) were associated with decreased $\mathrm{LVMI} / \mathrm{EDV}$, indicative of favourable LV remodelling and decrease E/e' ratio, a sign of decrease LA pressure. Higher levels of SB were associated with increased LVM. In the healthy subgroup, both MVPA and light PA were associated with greater LV myocardial mechanics, decrease LA pressure, and better RV function. SB was associated with 
Table 3 Multivariable linear regression for the association between MVPA and echocardiogram measures

\begin{tabular}{|c|c|c|c|}
\hline Outcome & Model 1 & Model 2 & Model 3 \\
\hline LVMI, $\mathrm{g} / \mathrm{m}^{2}$ & 0.0411 & 0.1318 & 0.1381 \\
\hline $\mathrm{LVMI}^{2.7} / \mathrm{EDV}$ & -0.0014 & -0.0006 & -0.0006 \\
\hline RWT, ratio & -0.0005 & -0.0001 & 0.0001 \\
\hline LAVI, mL/m² & $0.1707^{\star}$ & $0.1808^{\star}$ & $0.1792^{\star}$ \\
\hline $\mathrm{EF}, \%$ & 0.0578 & 0.0448 & 0.0389 \\
\hline $\mathrm{E} / \mathrm{e}^{\prime}$, ratio & 0.0037 & 0.0221 & 0.0259 \\
\hline GLS, \% & -0.069 & -0.0521 & -0.0539 \\
\hline GCS, $\%$ & $-0.2477^{\star}$ & $-0.2397^{\star}$ & $-0.2397^{\star}$ \\
\hline SV, mL & 0.3277 & 0.3177 & 0.3174 \\
\hline RV peak $S, \mathrm{~cm} / \mathrm{s}$ & 0.0589 & 0.0590 & 0.0615 \\
\hline RV FAC, \% & 0.0021 & 0.0019 & 0.0019 \\
\hline TRV Max, cm/s & 1.1367 & 1.1386 & 1.1597 \\
\hline TAPSE, cm & 0.0084 & 0.0082 & 0.0078 \\
\hline
\end{tabular}

M1: demographics (age and sex).

M2: M1+systolic blood pressure, antihypertensive med use.

M3: M2+other cardiovascular disease modifiable factors (diabetes mellitus, current smoking and high-density lipoprotein).

Values are per $10 \mathrm{~min} /$ day light MVPA.

${ }^{*} \mathrm{P}$ value $<0.01$

$E$, peak early diastolic transmitral inflow velocities; EDV, end diastolic volume; $\mathrm{e}^{\prime}$, average of mitral early diastolic septal and lateral annular velocities; EF, ejection fraction; GCS, global circumferential strain; GLS, global longitudinal strain; LAVI, left atrial volume index; LVMI, left ventricular mass index; M, model; MVPA, moderate to vigorous PA; RV, right ventricular; RV FAC, RV fractional area change; RWT, relative valve thickness; SV, stroke volume; TAPSE, tricuspid annular plane systolic excursion; TRV, tricuspid regurgitation velocity.

worsening LV myocardial mechanics, worsening LA pressure, and RV function.

Although increased PA has been associated with higher LVM, LA size and LV EDV in multiple prior studies, ${ }^{572425}$ to our knowledge, this is the first study to examine the association between accelerometry measured MVPA and improved LV myocardial mechanics. While there was no clinically significant association between LVEF and MVPA, LV GCS was significantly more favourable with MVPA. GCS reflects the change in length around the LV perimeter during systole as the myocardium shortens and the LV cavity circumference reduces. During incremental dynamic aerobic exercise, studies reported a progressive increase in GCS due to increased apical circumferential strain (ACS) with trivial effects on basal circumferential strain (BCS).$^{26}$ In contrast, GCS decreased during the isometric exercise with no effect on ACS and decreased BCS from rest. ${ }^{26}$ Therefore, exercise modality plays some role in GCS responses, and we did not have a granular breakdown of PA type. Further studies of global and region-specific circumferential strain during incremental exercise are lacking at present, and GCS responses during dynamic and static modalities remain incompletely understood.
Table 4 Multivariable linear regression for the association between sedentary behaviour and echocardiogram measures

\begin{tabular}{|c|c|c|c|}
\hline Outcome & Model 1 & Model 2 & Model 3 \\
\hline LVMI, $\mathrm{g} / \mathrm{m}^{2.7}$ & $0.0626^{\star}$ & 0.0485 & 0.0433 \\
\hline $\mathrm{LVMI}^{2.7} / \mathrm{EDV}$ & 0.0008 & 0.0006 & 0.0005 \\
\hline RWT, ratio & 0.0000 & -0.0002 & 0.0000 \\
\hline LAVI, $\mathrm{mL} / \mathrm{m}^{2}$ & -0.0066 & -0.0066 & -0.0035 \\
\hline $\mathrm{EF}, \%$ & -0.0025 & -0.0005 & 0.0025 \\
\hline $\mathrm{E} / \mathrm{e}^{\prime}$, ratio & 0.0144 & -0.0074 & -0.0341 \\
\hline $\mathrm{GLS}, \%$ & 0.0041 & 0.0035 & 0.0014 \\
\hline GCS, $\%$ & -0.0377 & -0.0390 & -0.0413 \\
\hline SV, mL & 0.0073 & -0.0039 & 0.0001 \\
\hline RV peak S, cm/s & 0.0090 & 0.0075 & 0.0068 \\
\hline RV FAC & 0.0001 & 0.0001 & 0.0002 \\
\hline TRV max, cm/s & -0.0457 & -0.0459 & -0.0330 \\
\hline TAPSE, cm & -0.0010 & -0.0011 & -0.0010 \\
\hline
\end{tabular}

M1: demographics (age and sex).

M2: M1+systolic blood pressure, antihypertensive med use. M3: M2+other cardiovascular disease modifiable factors (diabetes mellitus, current smoking and high-density lipoprotein).

Values are per $10 \mathrm{~min} /$ day of sedentary behaviour.

${ }^{*} P$ value $<0.01$.

$E$, peak early diastolic transmitral inflow velocities; EDV, end diastolic volume; $e^{\prime}$, average of mitral early diastolic septal and lateral annular velocities; EF, ejection fraction; GCS, global circumferential strain; GLS, global longitudinal strain; LAVI, left atrial volume index; LVMI, left ventricular mass index; M, model; $\mathrm{RV}$, right ventricular; RV FAC, RV fractional area change; RWT, relative valve thickness; SV, stroke vol; TAPSE, tricuspid annular plane systolic excursion; TRV, tricuspid regurgitation velocity.

Light PA was associated with better RV systolic function. Besides, higher quartiles of MVPA were also associated with better RV systolic function. Although these findings' implications are unclear, even small absolute changes in the very thin-walled $\mathrm{RV}$ in disease-free participants may have significant long-term effects. Aaron et al found that higher levels of both MVPA and intentional exercise were associated with larger RV stroke volume without a change in RVEF. ${ }^{27}$ Recent studies of patients with pulmonary arterial hypertension have shown that exercise training is safe and can improve exercise endurance, $6 \mathrm{~min}$ walk distance and quality of life. ${ }^{28}$ Recently, it has been noted that higher cardiorespiratory fitness (CRF) was associated with improved RV systolic function (TAPSE, RV S' wave) ${ }^{29}$ In addition, less decline in PA over time was associated with better RV systolic function. Structural and functional LV abnormalities may contribute to abnormalities in RV function. LV systolic or diastolic dysfunction leads to elevated LV end-diastolic pressure, which may cause pulmonary venous hypertension and high pulmonary artery systolic pressure, and RV systolic dysfunction. In addition, HF risk factors can adversely affect both RV and LV function such that subclinical abnormalities in RV function may precede and contribute to the 
development of HF, especially in HFpEF. Taken together, such exercise-induced adaptive changes in RV may be beneficial with potentially important implications for diseases characterised by pulmonary hypertension and $\mathrm{RV}$ dysfunction. These data may be useful in studying the $\mathrm{RV}$-specific benefits of exercise in at-risk populations.

Increases in LA size associated with higher CV fitness and MVPA have been described..$^{4-7}$ In the general population, however, increased LA size is associated with many pathological conditions, and it is usually the result of two processes, either increased volume or increased cardiac filling pressures. Interestingly, similar to our findings, the Cooper Clinic Longitudinal study observed no apparent association between diastolic dysfunction and LA size among high-fit individuals. ${ }^{4}$ Our findings and those reported by others suggest that LA dilation mechanisms may reflect physiology unrelated to diastolic function (ie, greater cardiac output) with higher PA. ${ }^{4-7}$

Recently Dallas Heart Study, which included 1368 participants (age, 49 years; $40 \%$ men, free of CVD), showed that objective measures of MVPA (assessed by accelerometer) were positively associated with more favourable LV structure and function parameters (assessed cardiac MRI), with better LV contractility, higher SV, lower arterial elastance, and LVH without myocardial injury, independent of traditional CVD risk factors. ${ }^{7}$ Vigorous PA was also positively associated with higher SV and lower effective arterial elastance measures and greater likelihood of both LVH without myocardial injury and malignant LVH. The moderate PA levels and SB were not associated with LV structure and function in the most adjusted analysis. Higher quartiles of MVPA and light PA were associated with favourable LV remodelling and improved diastolic function.

Improved diastolic function, increased LA size, improved LV mechanics (GCS), and RV systolic function further support that physiological adaptation with increased cardiac size characterised by normal cardiac morphology with a normal and enhanced cardiac function. This is similar to the recent Cooper Clinic Longitudinal study, which showed that higher cardiac fitness levels were independently associated with increased LA size, LV internal diameter at diastole, and decreased RWT and decreased E/e'. ${ }^{4}$ Similar to our study, there was no positive association between higher fitness and LVMI. This evidence taken together with our study confirms that staying active is more strongly associated with favourable measures of cardiac structure and function even at the level of light PA. Our improved LV strain, LV remodelling, LA volume, and improved diastolic function align with Dallas heart study results. ${ }^{7}$ These results further support the physiological benefits of long-term aerobic exercise and PA on cardiac structure and function. Importantly prior study also showed that only MVPA but not sedentary time were significantly associated with CRF levels.

While LVM, RV mass, LV volumes tend to decrease with bed rest or deconditioning. ${ }^{30}$ The chronic consequences of SB (increased obesity or obesity-related comorbidities, diabetes and hypertension) are generally associated with increased LVM and worse cardiac function. ${ }^{31-33}$ Thus, it is unclear whether and in which direction SB would be related to LV structure and function independently of PA, obesity and obesity-related comorbidities. A recent community-based study that included middle-aged adults free from overt clinical CV disease found that higher sedentary screen time was associated with increased LVMI, mass-to-volume ratio and RWT in Caucasians but not African Americans. ${ }^{34}$ Similarly, low fitness was independently associated with smaller heart size and concentric remodelling patterns in the Cooper Clinic Longitudinal Study. ${ }^{4}$

Taken together from ours and other studies, it has been noted that PA or higher CRF was associated with greater LV myocardial mechanics (LV strain), decreased LVMI/EDV (indicative of favourable $\mathrm{LV}$ remodelling), decrease $\mathrm{E} / \mathrm{e}^{\prime}$ ratio (a sign of decrease LA pressure), improved myocardial mechanics, lower arterial stiffness and improved RV function, which further supports the role of long-term aerobic exercise in preventing HF. ${ }^{29}{ }^{35-37}$ Besides, SB has been associated with higher HF risk and a greater burden of abnormalities in LV structure. Furthermore, low CRF also explained up to $40 \%$ of HF's high body mass index associated risk. ${ }^{35}$ This is very important with HFpEF since there is no proven benefit with pharmacological therapy in HFpEF except exercise training and calorie restriction and obesity is parallel with the HFpEF epidemic. ${ }^{38}$ While there is a large base of data that supports the CV benefits of PA and the detrimental effect of SB, these effects are broad and pleiotropic. PA is associated with various CV health benefits; decrease in BP, improved metabolic health, improved vascular function, decrease vascular stiffness, improved endothelial function, prevention of age-related cardiac remodelling, systolic and diastolic dysfunction, and adaptive pro-hypertrophic effect. ${ }^{4-73940}$

It is hypothesised that improved vascular function is due to reduced oxidative stress and subsequent improved endothelial dysfunction from PA. A separate study demonstrated that exercise could return the expected age-associated vascular tone to a more vasodilated state equivalent to that of younger subjects. ${ }^{40}$

\section{Strength and limitations}

The sizeable community-based sample, comprehensive assessment of PA (including SB and light PA) and echocardiographic measurements (including diastolic function, strain analysis, RV function) strengthen our investigations. Our study is also unique since this included an understudied high-risk population with a high prevalence of CV disease, risk factors and comorbidities. Most prior studies rely on the self-reported assessment of PA and SB. Using accelerometers to objectively quantify PA and SB reduces measurement error and allows for a more precise categorisation of the degree and amount of PA and SB.

Nonetheless, several limitations merit comment. The cross-sectional nature of this analysis prevents any causal inferences. Although we adjusted all models for multiple 
variables, the influence of residual confounding cannot be excluded. The accelerometry data were obtained prior to echocardiography, and that can result in underestimating the strength of the associations between MVPA and cardiac measurements (regression dilution bias). The hip-worn accelerometer is likely to underestimate certain activities such as bicycling and swimming (since they removed it when in the water). Additionally, accelerometry was assessed for only 1 week. While this is standard in the field, ${ }^{41}$ and others have shown it may reflect longer-term patterns of $\mathrm{PA}$ and $\mathrm{SB}$ among women, ${ }^{42}$ a longer measurement period would be an improvement.

Here we chose not to adjust the $\mathrm{p}$ value for multiple comparisons because each outcome was selected based on a priori hypothesis and a unique mechanism between exposure and each cardiac parameter. We were interested in controlling the type I error rate per the outcome of interest rather than per group of outcome variables. We only rejected the null hypothesis with a conservative $\mathrm{p}$ value of less than 0.01 . Nevertheless, the results should be interpreted cautiously and validated in other studies. Several of our findings may be considered hypothesisgenerating and warrant replication in subsequent studies. The sample size of ECHO-SOL is modest, limiting type II error. The type of PA (weight-training, cardio or isometric) may influence a different cardiac response (with GCS or other parameters). We did not have a PA-type breakdown for accelerometry data. Besides, accelerometry does not capture resistance training.

\section{CONCLUSION}

In a community-based population with multiple risk factors for developing LV dysfunction, both MVPA and light PA were associated with favourable cardiac mechanics after adjusting for major CV risk factors. Future studies of PA and cardiac function with a larger sample and a prospective longitudinal design are needed to assess cardiac function with clinical outcomes better.

\section{Author affiliations}

${ }^{1}$ Cardiology, Wake Forest Baptist Medical Center, Winston-Salem, North Carolina, USA

${ }^{2}$ Department of Family Medicine and Public Health, University of California San Diego, La Jolla, California, USA

${ }^{3}$ Department of Medicine, Epidemiology \& Population Health, Albert Einstein College of Medicine Department of Neurology, Bronx, New York, USA

${ }^{4}$ Department of Psychology, University of Miami, Coral Gables, Florida, USA

${ }^{5}$ Cardiovascular Medicine, University of Illinois Hospital and Health Sciences, Chicago, Illinois, USA

${ }^{6}$ Department of Epidemiology, Gillings School of Global Public Health, University of North Carolina - Chapel Hill, Chapel Hill, North Carolina, USA

Acknowledgements The authors acknowledge the investigators, the staff and the HCHS-SOL and ECHO-SOL participants for their dedication and commitment to the success of this study. Investigators website-http://www.cscc.unc.edu/hchs/.

Contributors All authors contributed to the design. AEB, BU, KS and CJR carried out the data collection and guarantees data integrity. KS performed statistical analyses. SP, RS, RK, PMV, QQ, NS, BEH, MD, MK and KRE reviewed the analysis, and $A E B$ and BU wrote the first draft. All authors contributed to revising and finalisation the manuscript. BU (corresponding author) guarantees all aspects of the reliability and freedom from bias of the data presented and their discussed interpretation.

Funding The Hispanic Community Health Study/Study of Latinos was carried out as a collaborative study supported by contracts from the National Institutes of Health (NIH) National Heart, Lung, and Blood Institute (NHLBI) to the University of North Carolina (N01-HC65233), University of Miami (N01-HC65234), Albert Einstein College of Medicine (N01-HC65235), Northwestern University (N01-HC65236) and San Diego State University (N01-HC65237). The following Institutes/Centres/Offices contribute to the HCHS/SOL through a transfer of funds to the NHLBI: National Institute on Minority Health and Health Disparities, National Institute on Deafness and Other Communication Disorders, National Institute of Dental and Craniofacial Research, National Institute of Diabetes and Digestive and Kidney Diseases, National Institute of Neurological Disorders and Stroke, NIH Institution-Office of Dietary Supplements. ECHO-SOL was supported by a grant from the National Heart, Lung, and Blood Institute (R01 HL104199, Epidemiologic Determinants of Cardiac Structure and Function among Hispanics: Carlos J Rodriguez, MD, MPH Principal Investigator)

Disclaimer The content is solely the responsibility of the authors and does not necessarily represent the official views of the NIH.

Competing interests None declared.

Patient consent for publication Not required.

Ethics approval Institutional Review Board approval was obtained at each participating institution, and all ECHO-SOL participants provided written consent.

Provenance and peer review Not commissioned; externally peer reviewed.

Data availability statement Data may be obtained from a third party and are not publicly available. All HCHS-SOL data will be included in a limited access database that will be available to the larger scientific public as part of the main HCHSSOL study database for public use. It will be encouraged that all use of data and publications from ancillary studies invite the participation of the ancillary study PI and coinvestigators.

Open access This is an open access article distributed in accordance with the Creative Commons Attribution Non Commercial (CC BY-NC 4.0) license, which permits others to distribute, remix, adapt, build upon this work non-commercially, and license their derivative works on different terms, provided the original work is properly cited, appropriate credit is given, any changes made indicated, and the use is non-commercial. See: http://creativecommons.org/licenses/by-nc/4.0/.

ORCID iD

Bharathi Upadhya http://orcid.org/0000-0001-7192-7995

\section{REFERENCES}

1 Whitfield GP, Carlson SA, Ussery EN, et al. Trends in Meeting Physical Activity Guidelines among Urban and Rural Dwelling Adults - United States, 2008-2017. MMWR Morb Mortal Wkly Rep 2019;68:513-8.

2 Summary health statistics for U.S. adults: National health interview survey. Available: https://ftp.cdc.gov/pub/Health_Statistics/NCHS/ NHIS/SHS/2018_SHS_Table_A-14.pdf

3 Du Y, Liu B, Sun Y, et al. Trends in adherence to the physical activity guidelines for Americans for aerobic activity and time spent on sedentary behavior among US adults, 2007 to 2016. JAMA Netw Open 2019;2:e197597.

4 Brinker SK, Pandey A, Ayers CR, et al. Association of cardiorespiratory fitness with left ventricular remodeling and diastolic function: the Cooper center longitudinal study. JACC Heart Fail 2014;2:238-46.

5 Turkbey EB, Jorgensen NW, Johnson WC, et al. Physical activity and physiological cardiac remodelling in a community setting: the multiethnic study of atherosclerosis (MESA). Heart 2010;96:42-8.

6 Andersson C, Lyass A, Larson MG, et al. Physical activity measured by Accelerometry and its associations with cardiac structure and vascular function in young and Middle-Aged adults. J Am Heart Assoc 2015;4:e001528.

7 Thangada ND, Patel KV, Peden B, et al. Cross-Sectional associations of objectively measured sedentary time, physical activity, and fitness with cardiac structure and function: findings from the Dallas heart study. J Am Heart Assoc 2021;10:e015601.

8 Nystoriak MA, Bhatnagar A. Cardiovascular effects and benefits of exercise. Front Cardiovasc Med 2018;28:135.

9 Qi Q, Strizich G, Merchant G, et al. Objectively measured sedentary time and cardiometabolic biomarkers in US Hispanic/Latino adults: 
the Hispanic Community Health Study/Study of Latinos (HCHS/ SOL). Circulation 2015;132:1560-9.

10 Kriska AM, Saremi A, Hanson RL, et al. Physical activity, obesity, and the incidence of type 2 diabetes in a high-risk population. Am J Epidemiol 2003;158:669-75.

11 Parker ED, Schmitz KH, Jacobs DR, et al. Physical activity in young adults and incident hypertension over 15 years of follow-up: the cardia study. Am J Public Health 2007;97:703-9.

12 Oktay AA, Rich JD, Shah SJ. The emerging epidemic of heart failure with preserved ejection fraction. Curr Heart Fail Rep 2013;10:401-10.

13 Rodriguez CJ, Allison M, Daviglus ML, et al. Status of cardiovascular disease and stroke in Hispanics/Latinos in the United States: a science Advisory from the American heart association. Circulation 2014;130:593-625.

14 Daviglus ML, Talavera GA, Avilés-Santa ML, et al. Prevalence of major cardiovascular risk factors and cardiovascular diseases among Hispanic/Latino individuals of diverse backgrounds in the United States. JAMA 2012;308:1775-84.

15 Lavange LM, Kalsbeek WD, Sorlie PD, et al. Sample design and cohort selection in the Hispanic Community Health Study/Study of Latinos. Ann Epidemiol 2010;20:642-9.

16 Rodriguez CJ, Dharod A, Allison MA, et al. Rationale and design of the Echocardiographic Study of Hispanics/Latinos (ECHO-SOL). Ethn Dis 2015;25:180-6.

17 Qureshi WT, Leigh JA, Swett K, et al. Comparison of echocardiographic measures in a Hispanic/Latino population with the 2005 and 2015 American Society of echocardiography reference limits (the echocardiographic study of Latinos). Circulation 2016;9:10.1161.

18 Evenson KR, Sotres-Alvarez D, Deng YU, et al. Accelerometer adherence and performance in a cohort study of US Hispanic adults. Medicine and science in sports and exercise 2015;47:725-34.

19 Colley RC, Tremblay MS. Moderate and vigorous physical activity intensity cut-points for the Actical accelerometer. J Sports Sci 2011;29:783-9.

20 Wong SL, Colley R, Connor Gorber S, et al. Actical accelerometer sedentary activity thresholds for adults. J Phys Act Health 2011;8:587-91.

21 Mehta H, Armstrong A, Swett K, et al. Burden of systolic and diastolic left ventricular dysfunction among Hispanics in the United States: insights from the echocardiographic study of Latinos. Circ Heart Fail 2016:9:e002733.

22 Lang RM, Badano LP, Mor-Avi V, et al. Recommendations for cardiac chamber quantification by echocardiography in adults: an update from the American Society of echocardiography and the European association of cardiovascular imaging. J Am Soc Echocardiogr 2015;28:1-39. e14.

23 Kaplan RC, Bangdiwala SI, Barnhart JM, et al. Smoking among U.S. Hispanic/Latino adults: the Hispanic community health Study/Study of Latinos. Am J Prev Med 2014;46:496-506.

24 Savage DD, Levy D, Dannenberg AL, et al. Association of echocardiographic left ventricular mass with body size, blood pressure and physical activity (the Framingham study). Am J Cardiol 1990;65:371-6.

25 Gardin JM, Wagenknecht LE, Anton-Culver H, et al. Relationship of cardiovascular risk factors to echocardiographic left ventricular mass in healthy young black and white adult men and women. The cardia study. Coronary artery risk development in young adults. Circulation 1995;92:380-7.

26 Beaumont A, Sculthorpe N, Hough J, et al. Global and regional left ventricular circumferential strain during incremental cycling and isometric knee extension exercise. Echocardiography 2018;35:1149-56.

27 Aaron CP, Tandri H, Barr RG, et al. Physical activity and right ventricular structure and function. The MESA-Right ventricle study. Am J Respir Crit Care Med 2011;183:396-404.

28 Mereles D, Ehlken N, Kreuscher S, et al. Exercise and respiratory training improve exercise capacity and quality of life in patients with severe chronic pulmonary hypertension. Circulation 2006;114:1482-9.

29 Patel KV, Metzinger M, Park B, et al. Longitudinal associations of fitness and obesity in young adulthood with right ventricular function and pulmonary artery systolic pressure in middle age: the cardia study. J Am Heart Assoc 2021;10:e016968.

30 Westby CM, Martin DS, Lee SMC, et al. Left ventricular remodeling during and after 60 days of sedentary head-down bed rest. $J$ Appl Physiol 2016;120:956-64.

31 Karason K, Wallentin I, Larsson B, et al. Effects of obesity and weight loss on left ventricular mass and relative wall thickness: survey and intervention study. BMJ 1997:315:912-6.

32 Levelt E, Mahmod M, Piechnik SK, et al. Relationship between left ventricular structural and metabolic remodeling in type 2 diabetes. Diabetes 2016:65:44-52.

33 Devereux RB, de Simone G, Ganau A, et al. Left ventricular hypertrophy and hypertension. Clin Exp Hypertens 1993;15:1025-32.

34 Gibbs BB, Reis JP, Schelbert EB, et al. Sedentary screen time and left ventricular structure and function: the CARDIA study. Med Sci Sports Exerc 2014;46:276-83.

35 Pandey A, Park B, Martens S, et al. Relationship of cardiorespiratory fitness and adiposity with left ventricular strain in middle-age adults (from the Dallas heart study). Am J Cardiol 2017;120:1405-9.

36 Pandey A, Allen NB, Ayers C, et al. Fitness in young adulthood and long-term cardiac structure and function: the CARDIA study. JACC Heart Fail 2017;5:347-55.

37 Pandey A, Cornwell WK, Willis B, et al. Body mass index and cardiorespiratory fitness in mid-life and risk of heart failure hospitalization in older age: findings from the Cooper center longitudinal study. JACC Heart Fail 2017:5:367-74.

38 Upadhya B, Haykowsky MJ, Kitzman DW. Therapy for heart failure with preserved ejection fraction: current status, unique challenges, and future directions. Heart Fail Rev 2018;23:609-29.

39 Santos-Parker JR, LaRocca TJ, Seals DR. Aerobic exercise and other healthy lifestyle factors that influence vascular aging. Adv Physiol Educ 2014;38:296-307.

40 Van Guilder GP, Westby CM, Greiner JJ, et al. Endothelin-1 vasoconstrictor tone increases with age in healthy men but can be reduced by regular aerobic exercise. Hypertension 2007;50:403-9.

41 Vetrovsky T, Clark CCT, Bisi MC, et al. Advances in accelerometry for cardiovascular patients: a systematic review with practical recommendations. ESC Heart Fail 2020;7:2021-31.

42 Keadle SK, Shiroma EJ, Kamada M, et al. Reproducibility of Accelerometer-Assessed physical activity and sedentary time. Am J Prev Med 2017;52:541-8. 